\title{
Procesos de inclusión social de las personas sin hogar en la ciudad de Barcelona: relatos de vida y acompañamiento social*
}

\section{María Virginia Matulič Domandzič}

Departamento de Trabajo Social y Servicios Sociales, Universidad de Barcelona. mmatulic@ub.edu

\begin{abstract}
Artikulu hau Bartzelonako hiriko etxegabeko pertsonen gizarteratze sozialeko prozesuak: Bizikontakizunak eta lagun-egite soziala doktoregotesiaren fruitu bat da, 2016 urtean Bartzelonako Unibertsitateko (UB) Gizarte Langintzako Departamentuan defendatutakoa. Aipatutako lanak zerikusia izan zuen UBko Hezkuntza Fakultatearen Hezkuntza eta Gizartea Doktorego Programarekin. Ikerketaren xedea Bartzelonako etxegabekoen gizarteratzera-prozesuak erraztu eta mugatzen dituzten alderdiak ezagutzea zen, haien esanaren eta lagundutako gizarte-profesionalen esanaren bitartez. Alderdi lagungarri nagusi gisara, erakundeekin duten atxikimendua eta etxegabekoek igarotzen dituzten prozesu konplexuetan zehar profesionalek egindako lagun-egite soziala nabarmentzen dira.
\end{abstract}

\section{GAKO-HITZAK:}

Etxegabeko pertsonak, gizarteratze-prozesuak, gizarte-zerbitzuak, gizarte-politikak, lagun-egite soziala.
Este artículo es el fruto de la tesis doctoral Procesos de inclusión social de las personas sin hogar en la ciudad de Barcelona: Relatos de vida y acompañamiento social defendida en el Departamento de Trabajo Social de la Universidad de Barcelona (UB) en el año 2016. Dicho trabajo de investigación estaba vinculado al Programa de Doctorado Educación y Sociedad de la Facultad de Educación de la UB.

Desde esta investigación se planteaba conocer los elementos que favorecen y limitan los procesos de inclusión social de las personas sin hogar en Barcelona, a través de la voz de sus protagonistas y de los profesionales sociales que los acompañan. Destacan como principales elementos favorecedores la vinculación a las entidades y el acompañamiento social realizado por los profesionales a lo largo de los complejos procesos por los que transitan las personas sin hogar.

\section{Palabras Clave:}

personas sin hogar, procesos de inclusión, servicios sociales, políticas sociales, acompañamiento social. 


\section{Introducción}

Las personas sin hogar representan el paradigma extremo de la pobreza y la exclusión social, en el que se conjuga una serie de factores combinados (estructurales, relacionales e individuales) que se materializan en vivir en la calle. En la actualidad este fenómeno continúa impregnado de un imaginario social estigmatizador y excluyente que pone en duda la propia condición de ciudadanos de las personas que lo padecen (Cabrera, 2008). La exclusión social que presentan las personas sin hogar nos habla de procesos o trayectorias de vida en que las personas se encuentran desvinculadas o se van desvinculando progresivamente de los elementos que garantizan la integración en nuestra sociedad, como son el mercado, las políticas de redistribución y la reciprocidad social y comunitaria. La ausencia de toda participación en la vida productiva y social genera el proceso de desafiliación social, término acuñado por Castel (1991).

El trabajo social como disciplina científica aporta continuas mejoras a la sociedad, porque a través de la intervención en contextos y con personas que se encuentran en situaciones de exclusión social, se empoderan procesos y se reducen factores generadores de desigualdad social. Nuestra disciplina tiene una amplia trayectoria de intervención en situaciones de exclusión social incorporando una mirada micro y macrosocial a los fenómenos en los que interviene.

La ciudad de Barcelona cuenta con una red de entidades y organizaciones público-privadas que dirigen su atención a las situaciones de sinhogarismo. Desde 1985 el ayuntamiento de la ciudad ha implementado programas municipales y actuaciones que se han ido ampliando y renovando en función de las necesidades y de nuevos planteamientos sobre la temática. Desde el 2014 se aplican dos modelos de intervención, el de escalera o transición (en el que la persona después de un periodo de intervención social pasa por diversos recursos de inclusión social hasta conseguir al final el acceso a una vivienda) y el modelo Housing First como propuesta de intervención centrada en el derecho a la vivienda.

El objetivo principal planteado en esta investigación era analizar los procesos de inclusión de las personas sin hogar en la ciudad de Barcelona a través de la voz de sus propios protagonistas, pudiendo identificar los elementos que favorecen y dificultan dichos procesos. También se planteaban conocer el conjunto de actuaciones profesionales que intervienen en dichos procesos de inclusión social. La metodología aplicada de carácter cualitativo pretendía visualizar y profundizar en los procesos de inclusión social a través de doce relatos de vida a hombres y mujeres y de veintisiete entrevistas en profundidad realizadas a profesionales sociales que las acompañan.

Esta investigación es novedosa porque aporta un mayor conocimiento de los procesos de inclusión social de las personas sin hogar y de los elementos fundamentales vinculados al espacio relacional entre acompañados y acompañantes. Los resultados apuntan que los factores que intervienen en los procesos de inclusión social de las personas sin hogar se vinculan a factores de tipo estructural (entre los que destacan la articulación de programas y actuaciones orientadas al alojamiento, a la formación y a la inserción laboral), institucional (donde toma protagonismo el acompañamiento social proactivo, intensivo desplegado por los profesionales sociales), relacional (centradas en los apoyos familiares y sociales) y personal (donde tienen una importante incidencia las capacidades resilientes de las personas que transitan por estos difíciles procesos). Estos resultados coinciden con lo planteado por diversos autores (Cabrera, 2008; Tezanos, 2004; Renes et al., 2007; Roca, 2006; Subirats, 2004; Vidal, 2010) en relación con los factores protectores frente a las situaciones de sinhogarismo.

Este trabajo de investigación se divide en tres partes: el marco teórico, la metodología, los resultados y análisis de las dimensiones estudiadas. Finalmente se presentan las conclusiones y recomendaciones, así como las referencias bibliográficas utilizadas.

\section{Marco teórico}

La dimensión teórica de esta investigación consta de cuatro capítulos donde se realiza una introducción a los conceptos vinculados con la exclusión e inclusión social, a las causas y procesos por los que transitan las personas sin hogar, a los dispositivos articulados desde la red pública privada de la ciudad y a los acompañamientos realizados por los trabajadores sociales.

El concepto de exclusión social se utiliza a finales de la década del siglo XX para referirse a las diversas situaciones de privación presentes en las sociedades europeas. Castel (1997) denomina a este proceso desafiliación social, debido a que en él se conjugan diversos elementos negativos que ocasionan la ausencia de toda participación en una actividad productiva y en un aislamiento relacional. Dicha desafiliación va más allá de la idea de privación económica e incorpora la privación de derechos desde la misma concepción de la ciudadanía (Subirats, 2004). Tal como afirman diversos autores (Beck, 1998; Bauman, 2000), las fronteras de la exclusión son hoy altamente permeables, móviles y fluidas y sus riesgos son cada vez más amplios.

Los procesos de desvinculación o desafiliación social que presentan las personas sin hogar están asociados a diversos factores (de tipo estructural, relacional e individual) y responden a procesos y trayectorias vitales de variada etiología. En la actualidad existe un amplio consenso entre los estudiosos a determinar que no hay una única situación, sino la convergencia e interacción entre diversos factores donde la exclusión residencial toma 
un protagonismo primordial (Cabrera, 2008; Muñoz y Vázquez, 1998).

Según datos de la Diagnosis 2013 (Sales, 2013), el número de personas sin hogar en la ciudad de Barcelona era de $\mathbf{2 . 9 1 6}$ personas (de las que 870 eran personas sin techo y 1.451 estaban alojadas en centros residenciales). Con relación al género, el $90 \%$ son hombres; en cuanto a la edad, el 47 $\%$ tiene entre 30 y 44 años; y con respecto a la nacionalidad, el $43 \%$ era de nacionalidad española, el 30 \% de la Unión Europea y el 26,9 \% extranjera no comunitaria).

Tal como afirman diversos autores (Cabrera, 2008; Tezanos, 2004; Subirats, 2004), en el plano de la integración o protección intervienen cinco ámbitos o dimensiones: estructural, institucional, relacional, personal y de discriminación (atravesados por cuatro ejes: género, edad, etnia y discapacidad) que conforman las diferencias fundamentales en los procesos de integración por los que atraviesan las personas a lo largo de sus trayectorias vitales. Estos factores conjugados permiten hablar de la necesidad de abordar la inclusión social de las personas sin hogar desde una perspectiva integral. En este sentido, los recursos sociales deben orientarse a la idea de proceso en el que se conjugan diversas áreas interrelacionadas que están incidiendo en su evolución (Cabrera, 2008).

La ciudad de Barcelona cuenta desde el año 1985 con un Programa Municipal d'atenció social a Persones Sense Sostre que atiende las necesidades que presentan las personas en sus diversas fases de desvinculación social (inicial, avanzada y consolidada). En 2005 se consolida un modelo de cooperación entre el sistema público y la iniciativa social a partir de la configuración de la Xarxa d'atenció a Persones Sense Sostre en el marco del Acord Ciutadà per una Barcelona Inclusiva ${ }^{1}$ (Programa Municipal d'atenció a Persones Sense Sostre, 2007) en el que participan más de treinta entidades. La lógica de atención aplicada se centra en el modelo de escala o transición, que se caracteriza por ser gradual y procesual tanto en la provisión de recursos (atención a primeras necesidades, alojamientos temporales breves, recursos intermedios hasta la adjudicación de recursos finalistas) como en la intervención social realizada.

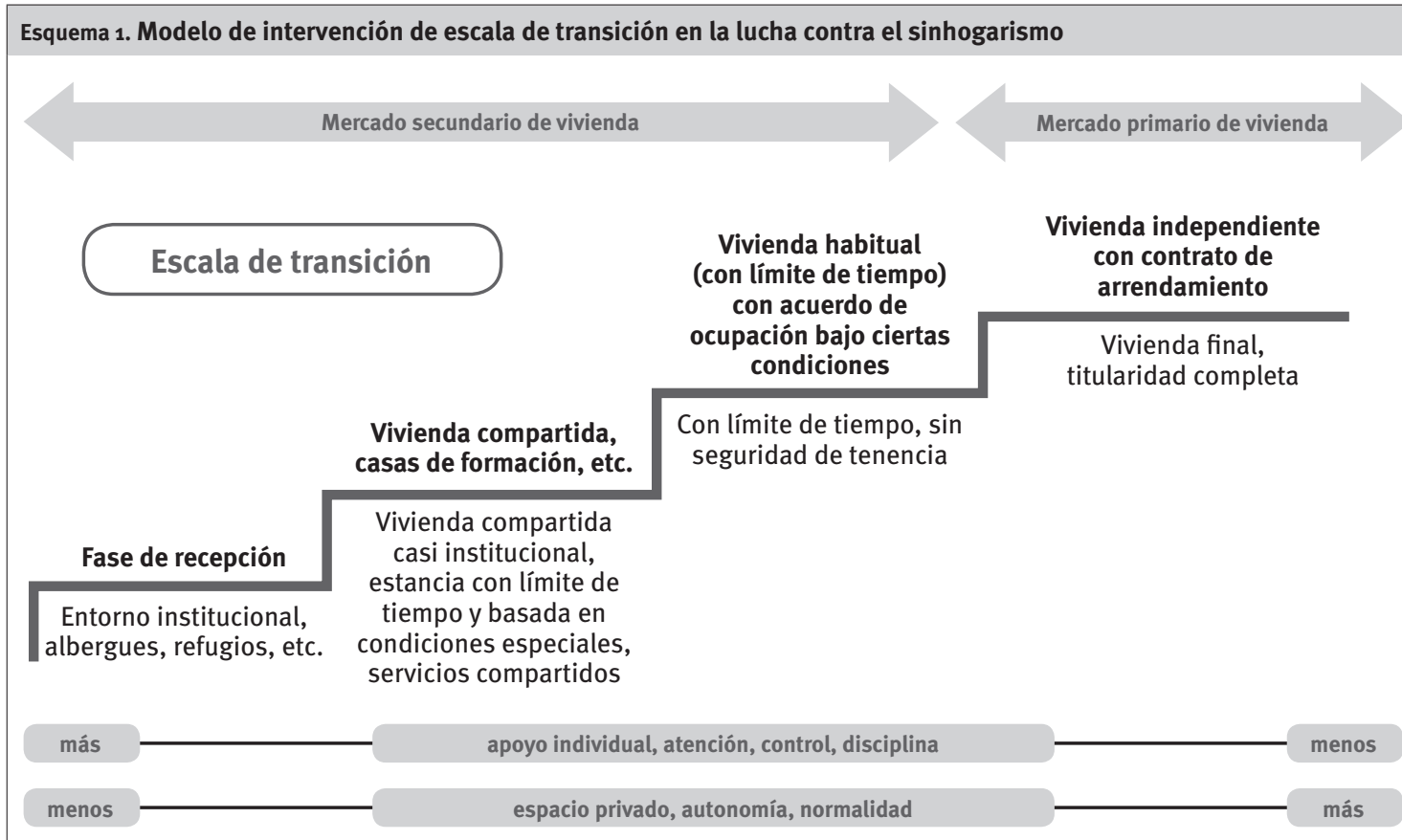

Fuente: Citado en Busch-Geertsema (2013: 16) y traducido por la autora.

${ }^{1}$ El Acuerdo Ciudadano por una Barcelona Inclusiva es el espacio de participación, de cooperación público-privada y de acción conjunta entre instituciones y organizaciones de la ciudad que trabajan para construir una Barcelona más inclusiva y con mayor calidad de vida para todas las personas. En definitiva, es un espacio compartido en tre el gobierno de la ciudad y la sociedad civil para la inclusión social. La finalidad del Acuerdo es incrementar el capital social de la ciudad, es decir, su capacidad de organización y de actuación conjunta. Y lo realiza mediante la promoción de la cooperación y de la articulación de redes de acción para avanzar en la construcción plural y democrática de un bien común primordial: la inclusión social. Participan unas 600 entidades de toda la ciudad (http://www.bcn.cat/barcelonainclusiva/es). 


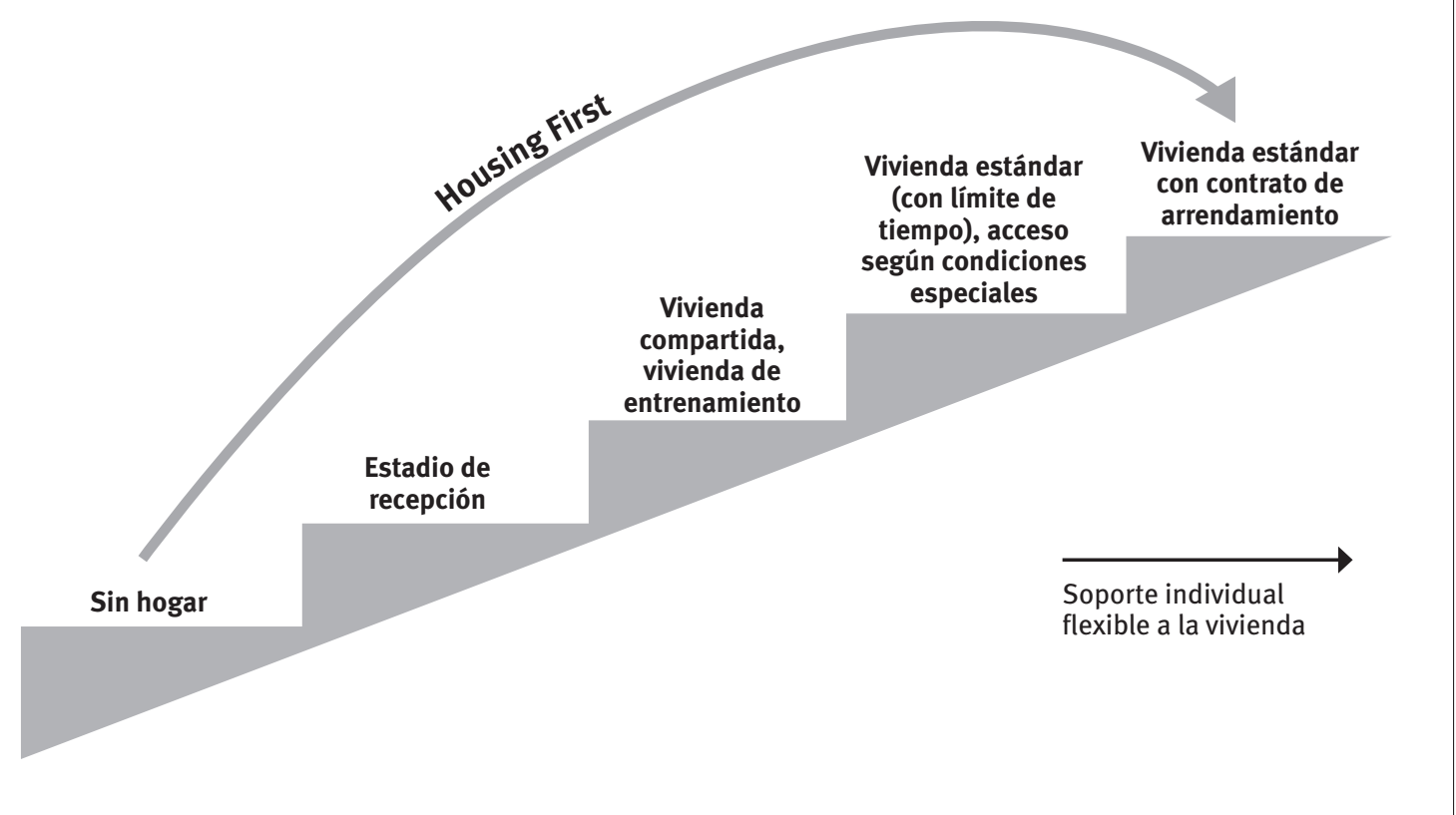

Fuente: Citado en Busch-Geertsema (2013: 17) y traducido por la autora.

Este modelo es el que se utiliza en la actualidad en la mayoría de los países europeos. Durante el año 2014, el Ayuntamiento de la ciudad apuesta por la incorporación del modelo Housing First ${ }^{2}$ en la ciudad.

Dichas actuaciones se implementan siguiendo las directrices de diversos documentos y organizaciones nacionales y europeas sobre el sinhogarismo (EAPN, 2013; European Comission, 2013; Feantsa, 2013; Fepsh, 2013; Sales, 2103; Uribe, 2014).

Las tres áreas de intervención dirigidas a las personas sin hogar se centran en la prevención, la satisfacción de necesidades básicas y la recuperación e inserción social (Cabrera, 2008). Las bases fundamentales en la que se articulan las intervenciones sociales en cada una de estas áreas son la proximidad ${ }^{3}$ y el acompañamiento social. A través de la relación de proximidad establecida con las personas en situación de exclusión social se despliega la metodología de intervención basada en el acompañamiento social. Tal y como definen Funes y Raya (2001), acompañar es avanzar "al lado de”, es compartir un proyecto común a lo largo de

${ }^{2}$ El modelo Housing First se centra en el conjunto de estrategias conocidas de housing-led, que debe su nombre a un programa iniciado en Estados Unidos por la organización Beyond the Shelter en 1988. Este modelo centra la atención en la vivienda primero, ofreciendo posteriormente el apoyo a través de equipos de soporte, a diferencia el modelo de escala de transición en que la vivienda es el último paso del proceso.

${ }^{3}$ La intervención de proximidad se asocia en España al trabajo de calle o en medio abierto que se aplica para trabajar diversas situaciones de dificultad en los entornos más habituales donde se encuentran las personas o grupos atendidos. La calle se convierte en un espacio educativo porque se pueden encontrar con los iguales y pueden ejercer maneras de ser propias de ellos mismos (Funes, 2001). los itinerarios de incorporación social, de forma complementaria al trabajo comunitario y a otro tipo de derechos sociales, garantía de ingresos y recursos de apoyo (Raya y Caparrós, 2014: 83).

Existen diferentes formas de acompañamiento: el acompañamiento social, el educativo y el terapéutico. A estas tres categorías también se debe añadir una cuarta, de tipo complementario, pero de gran relevancia en las situaciones de sinhogarismo, el acompañamiento entre recursos, dispositivos y servicios (Funes y Alonso, 2009: 30). En las situaciones de exclusión extrema se despliegan los cuatro tipos de acompañamientos que se relacionan con las intervenciones socioeducativas y socioterapéuticas.

Las fases del acompañamiento social se componen de tres momentos claves: construir la relación, el compromiso de acompañamiento a través del plan de trabajo compartido y la participación a través de la ciudadanía inclusiva. A través del compromiso de acompañamiento se despliegan diversos métodos de intervención (individual, grupal y comunitario) de forma interrelacionada y en función de las necesidades y potencialidades de las personas acompañadas. Desde la perspectiva individual con personas sin hogar la gestión de caso es el abordaje más utilizado (Eransus, 2004; Raya y Caparrós, 2014). Las técnicas grupales y comunitarias son utilizadas en menor medida con las personas sin hogar. El documento de la Comisión Europea (2013) afirma que los programas grupales pueden proporcionar actividades significativas para las personas que experimentan o han experimentado una situación de sinhogarismo. 
A partir del reconocimiento de derechos, la pertenencia a una comunidad y la participación en la vida social, económica y política, las personas pasarán de destinatarios a actores. La ciudadanía inclusiva da un paso más, implica el reconocimiento de derechos a todas las personas en igualdad de condiciones. Para trabajar de manera empoderadora, es necesario que los profesionales de las entidades destinadas a las personas sin hogar trabajen desde un enfoque holístico, es decir, desde las siguientes tres dimensiones: respetar los derechos y los principios de trabajo, la provisión de recursos y la creación de oportunidades (Feantsa, 2009: 6). En este sentido, tal como nos indica Vidal (2009: 413) necesitamos desarrollar un trabajo social narrativo que incorpore en la metodología de intervención social la activación de los significados y relatos como recursos de empoderamiento de los excluidos y el cambio social.

Los profesionales que intervienen en las entidades públicas y privadas de la Xarxa d'Atenció a Persones Sense Llar de Barcelona (Xapsll, Red de Atención a Personas sin Hogar) proceden de diversas disciplinas sociales, destacando mayoritariamente trabajadores sociales y educadores sociales. Sus funciones varían en relación con las finalidades del servicio y a las características de cada organización. Una de las variables a tener en cuenta con relación al tipo de intervención que realizan los profesionales son los contextos donde desarrollan su actividad (pudiendo ser en espacios abiertos o cerrados) y a los objetivos de su intervención (prevención, detección, tratamiento, rehabilitación y promoción de la ciudadanía).

\section{La metodología}

En esta investigación se ha optado por la utilización de una metodología cualitativa. Según Busquets, los métodos cualitativos buscan una mediación precisa de la realidad social y una comprensión más rica y compleja del fenómeno que se quiere estudiar (Busquets, et al, 2006).

Las preguntas y objetivos planteados en esta investigación tienen dos finalidades: identificar y comprender los procesos de inclusión social realizados por las personas sin hogar, y conocer y analizar los acompañamientos sociales realizados por los trabajadores sociales. A través del muestreo intencional utilizado se pretende que los sujetos entrevistados sean los más relevantes y pertinentes para el objeto de estudio (Ruiz, 2007). En esta investigación se seleccionaron siete entidades sociales de amplia trayectoria en la ciudad 4 : Arrels Fundació, Sant Joan de Déu, Serveis Socials, Centre d'acolliment residencial Can Planas, Centre Llar de Pau, Fundació Mambré, Obra Social Santa Lluïsa de Marillac y Progess S.L.

${ }^{4}$ Arrels Fundació fue creada en 1987, Sant Joan de Déu en el año 1979, Can Planas en 1999, Llar de Pau en 1991 y Santa Lluïsa de Marillac en el año 1983.
Los criterios de inclusión utilizados para las personas sin hogar se han basado en que estén vinculadas a las entidades participantes y se encuentren transitando por diversos procesos de inclusión social. Y para los profesionales, que tengan una experiencia de cinco a diez años y se encuentren acompañando diversos procesos de inclusión social desde dichas entidades.

Las técnicas utilizadas en esta investigación han sido la observación participante, los relatos de vida y las entrevistas en profundidad. El total de entrevistas realizadas han sido 49 (doce relatos de vida a mujeres y hombres sin hogar, 27 entrevistas en profundidad a profesionales sociales (trece a trabajadores sociales) y diez entrevistas exploratorias a directores, coordinadores y responsables de las diversas organizaciones. En la Tabla 1 vemos las entrevistas realizadas en esta investigación.

Tabla 1. Entrevistas realizadas

\begin{tabular}{|c|c|c|}
\hline $\begin{array}{l}\text { Técnicas de recogida de } \\
\text { información }\end{array}$ & $\begin{array}{l}\text { Guías de recogida de } \\
\text { información }\end{array}$ & $\begin{array}{l}\text { Número } \\
\text { total }\end{array}$ \\
\hline \multirow{2}{*}{$\begin{array}{l}\text { Relatos de vida de las } \\
\text { personas sin hogar } \\
\text { atendidas desde las } \\
\text { entidades de la XAPSLL }\end{array}$} & $\begin{array}{l}\text { Relatos de vida a hombres } \\
\text { atendidos desde la XAPSLL }\end{array}$ & 6 \\
\hline & $\begin{array}{l}\text { Relatos de vida a mujeres } \\
\text { atendidas desde la XAPSLL }\end{array}$ & 6 \\
\hline $\begin{array}{l}\text { Entrevistas exploratorias } \\
\text { y de seguimiento } \\
\text { a responsables del } \\
\text { ayuntamiento }\end{array}$ & $\begin{array}{l}\text { Entrevistas exploratorias } \\
\text { y de seguimiento } \\
\text { a responsables de } \\
\text { departamentos o unidades } \\
\text { del Ayuntamiento de } \\
\text { Barcelona }\end{array}$ & 3 \\
\hline \multirow{2}{*}{$\begin{array}{l}\text { Entrevistas en } \\
\text { profundidad a } \\
\text { responsables y } \\
\text { profesionales de las } \\
\text { entidades de la XAPSLL }\end{array}$} & $\begin{array}{l}\text { Entrevistas exploratorias } \\
\text { a responsables de las } \\
\text { entidades de la XAPSLL }\end{array}$ & 7 \\
\hline & $\begin{array}{l}\text { Entrevistas en profundidad } \\
\text { a profesionales de las } \\
\text { entidades de la XAPSLL }\end{array}$ & 27 \\
\hline \multicolumn{2}{|l|}{ Total entrevistas realizadas } & 49 \\
\hline
\end{tabular}

*XAPSLL: Xarxa d’Atenció a Persones Sense Llar (Red de Atención a Personas sin Hogar).

Las categorías planteadas en la dimensión de estudio de las personas sin hogar introducen la comprensión de los procesos de inclusión social a través de las trayectorias vitales y de las capacidades de las personas (factores que favorecen, redes de apoyo, vinculación con las entidades y profesionales y los cambios significativos narrados por las personas, así como sus expectativas de futuro), pudiendo partir de una mirada retrospectiva con relación a las causas de la exclusión social (incidiendo en los sucesos traumáticos y en factores que han dificultado procesos generadores de mejora).

Las categorías planteadas en la dimensión de los profesionales sociales introducen la mirada en los procesos de ayuda desplegados desde el acompañamiento social con personas en situación de calle (centrando la atención en el tipo de acompañamiento y en los modelos teóricos y técnicas utilizados por los trabajadores sociales). También se introduce la categoría sobre los factores que favorecen y dificultan los procesos (que también 


\begin{tabular}{|c|c|c|}
\hline Dimensiones & Categorías & Descripción \\
\hline \multirow{5}{*}{ Las personas sin hogar } & Sucesos traumáticos en el ciclo vital* & $\begin{array}{l}\text { Experiencias de carácter personal que han sido importantes } \\
\text { para el sujeto. }\end{array}$ \\
\hline & Las redes de apoyo & Los soportes sociales con los que cuenta la persona. \\
\hline & $\begin{array}{l}\text { La vinculación con las entidades y } \\
\text { profesionales }\end{array}$ & Proceso de vinculación, compromiso y participación. \\
\hline & Factores que favorecen y dificultan & $\begin{array}{l}\text { Factores que posibilitan (capacidades) y factores que } \\
\text { limitan (dificultades) los procesos de cambio identificados } \\
\text { por las personas. }\end{array}$ \\
\hline & Las expectativas de futuro & Las expectativas o retos planteados por las personas. \\
\hline \multirow{4}{*}{ Los profesionales sociales } & $\begin{array}{l}\text { Factores que favorecen y dificultan los } \\
\text { procesos }\end{array}$ & $\begin{array}{l}\text { Factores identificados por los profesionales que posibilitan } \\
\text { o limitan los procesos de cambios. }\end{array}$ \\
\hline & Modelos y técnicas de intervención & Modelos y técnicas aplicados en la intervención social. \\
\hline & $\begin{array}{l}\text { Acompañamiento social realizado por los } \\
\text { profesionales sociales }\end{array}$ & $\begin{array}{l}\text { Elementos destacables del acompañamiento social } \\
\text { realizado en la intervención social. }\end{array}$ \\
\hline & $\begin{array}{l}\text { Retos pendientes en la atención a las } \\
\text { personas sin hogar en la ciudad de } \\
\text { Barcelona. }\end{array}$ & $\begin{array}{l}\text { Las propuestas de futuro en relación con la intervención } \\
\text { con personas sin hogar en la ciudad de Barcelona. }\end{array}$ \\
\hline
\end{tabular}

* a) Sucesos traumáticos durante la infancia: abandono, internamiento, pérdida de alguno de los progenitores, malos tratos, padres o referentes adultos con problemáticas de salud mental o adicciones.

b) Sucesos traumáticos durante la adolescencia y juventud: pérdida de los progenitores, internamiento, fuga o abandono del hogar durante la adolescencia, consumo de tóxicos, problemas de salud, pérdida o separación de la pareja, pérdida del trabajo, la situación de estar en la calle. c) Sucesos traumáticos durante la vida adulta: pérdida de familiares, separaciones/divorcios, soledad, pérdida del trabajo, problemas de salud, la situación de estar en la calle.

aparece en la dimensión de las personas sin hogar) para ampliar su comprensión desde las dos miradas. Y, finalmente, se introduce una categoría orientada hacia el futuro con relación a los retos pendientes en la atención a las personas sin hogar en la ciudad de Barcelona. En el Cuadro 1 se detallan las dimensiones y categorías de análisis.

Debido a la temática y a las difíciles situaciones tratadas, esta tesis se enmarca en la dimensión de las investigaciones de naturaleza sensibles (Sieber y Stanley 1988). Los temas tratados con los entrevistados a lo largo del estudio se ocupan de áreas socialmente vulnerables que están cargadas de un gran potencial emocional; por ello, la investigadora además de establecer un vínculo previo con las personas entrevistadas, debía respetar los ritmos o momentos en que la persona se encontraba para explicar su historia.

\section{Los resultados}

Los resultados de esta tesis se agrupan en dos grandes apartados que se corresponden con las dimensiones de las personas sin hogar denominado Transitando procesos de inclusión y de los profesionales denominado Acompañando procesos de inclusión.

\subsection{Transitando procesos de inclusión}

\subsubsection{Los sucesos traumáticos y las redes de apoyo}

Los relatos de las personas sin hogar presentan diversos sucesos traumáticos a lo largo de sus ciclos vitales. Ocho de los doce relatos manifiestan haber pasado por varios de estos sucesos (los primeros durante la infancia y adolescencia y los segundos durante su vida adulta). Estos relatos son los que presentan un mayor número de sucesos impactantes a lo largo de sus trayectorias vitales (entre cinco y siete). Diversos autores (Muñoz et al., 2004; Muñoz et al., 2005; Vázquez et al., 2011) afirman que los sucesos acaecidos durante la infancia que pueden tener una especial incidencia en las situaciones de sinhogarismo son la institucionalización y el abandono, haber padecido malos tratos o la muerte de familiares en esta etapa de la vida.

“¿Recuerdos de la infancia? Pocos y no buenos, cómo explicarte... Yo he pasado mucho, pero mucho, con tres años fui violada y no se lo pude explicar a nadie, ni a mi madre, hasta que tuve 30 años. Y bueno, ahora lo explico porque me hace falta. Y más tarde, con 13 años ya me tiré a las drogas..." (Eg).

La ausencia de apego con figuras parentales en etapas tempranas de la vida ocasiona importantes dificultades relacionales (aislamiento social, relaciones conflictivas o inestables) y emocionales (autoestima baja, culpabilización o aparición de trastornos mentales) en la vida adulta (Bericat, 2000; Howe, 1997; Muñoz, 2004). La variable de género condiciona las experiencias vitales (Escudero, 2003; Morata, Morante y Trujillo, 2010) produciendo una mayor discriminación generada por factores estructurales (relacionadas con el acceso al trabajo y el sostenimiento económico), relacionales (que inciden en el rol y en las responsabilidades familiares otorgadas a las mujeres) y personales (vinculadas con altos niveles de victimización producidos por factores asociados a la salud mental, adicciones y violencia doméstica). 
Quedarse en la calle en las etapas adultas (madurez o vejez) está relacionado, en primer lugar, con factores estructurales (pérdida o dificultades de mantenimiento de la vivienda debido a la pérdida del empleo o a la carencia de prestaciones sociales); muy vinculado a factores relacionales (debilidad o ruptura de apoyos sociales); y asociado con problemas de salud mental que refuerzan el aislamiento y la desvinculación social (Anderson y Christian, 2003; Sales, 2013).

Los relatos de las personas sin hogar que se presentan en esta investigación mantienen una relación favorable con sus familiares. Ocho de las doce historias presentan vinculaciones de diferente intensidad con sus familias de origen (cinco tienen una relación continuada o estable y tres de ellas mantienen relaciones débiles e intermitentes). Disponer de redes sociales supone un indicador importante en los procesos de inclusión de las personas que transitan por una situación sin hogar. La ausencia de apoyos sociales aumenta las probabilidades de recaídas o cronificación en las situaciones de sinhogarismo (Sarasa y Sales, 2009), así como recuperar vínculos y relaciones significativas implica generar procesos de reconocimiento individual y social de efectos relevantes para estas personas.

\footnotetext{
“Con mi hermana empezó lo del VIH, con 23 años, y bueno, me costó mucho que no lo aceptara. Fue un golpe grande para mí decírselo a mi hermana mayor y que no lo aceptara. Entonces me tiré doce años sin hablarme con ella. Y ahora, junto con Juan, es la que me más me está ayudando a que esté saliendo adelante. Yo estoy aquí por ellos, y debo recuperarme porque se los debo" (Eg).
}

En relación con la vinculación con los profesionales, las personas entrevistadas manifiestan haberse sentido "respetadas y reconocidas", resaltando como determinante en la relación establecida "la calidad humana” y "estar disponibles" cuando era necesario e importante para ellas.
"Me han ayudado en todos los sitios donde he estado, pero la ayuda era puntual y volvía a recorrer lugares donde me volvían a ayudar. Aunque es aquí donde siento que realmente me han ayudado más, porque se preocupan por mí, tienen mucha paciencia conmigo cuando me dan esos prontos y no me dejan solo" (E12).

Otro punto de vital importancia manifestado por las personas entrevistadas es la seguridad, no solo material y económica, sino también emocional. Tal como afirman diversos autores (Declerck, 2001; García Roca, 2006) la incertidumbre en las que se encuentran inmersas estas personas las convierte en náufragos de su propio destino. En este sentido, ofrecer seguridad a través de un acompañamiento continuado y sostenido en el tiempo genera estabilidad emocional y abre nuevas perspectivas de cambios. Otros de los elementos valorados positivamente por las personas entrevistadas se relacionan con el apoyo ofrecido por los profesionales en momentos claves del proceso. Este soporte proporcionado en momentos claves de su situación ha facilitado la creación de nuevas oportunidades. En relación con los factores negativos de los profesionales, destacan la acción de dirigir y ejercer el control.

Otro de los aspectos relevantes destacados por las personas es la participación en diversos espacios de la entidad o fuera de ella. Diversos documentos (Feantsa, 2005; Fepsh, 2013; Comisión Europea, 2013) inciden en la importancia de la participación de las personas sin hogar en actividades y programas vinculados a las entidades.

Los relatos destacan como elementos negativos en relación con las entidades los relacionados con su configuración y normativas. Diversos autores (Cabrera, 1998; Vázquez et al., 2011; MorenoMárquez, 2012) apuntan que determinados servicios temporales, concretamente los albergues, son valorados negativamente por las personas sin hogar debido a aspectos relacionados con las relaciones entre las personas atendidas, el escaso nivel de intimidad y la inadecuación en sus horarios.

\subsubsection{Los factores que favorecen y dificultan los procesos de inclusión social}

Los factores de integración o inclusión pasan por cuatro ámbitos fundamentales: estructural, institucional, relacional y personal (Cabrera, 2008; Subirats, 2004; Tezanos, 2004). Los factores que favorecen y dificultan los procesos de inclusión social se vinculan a las tres fases en las que se encontraban las personas entrevistadas: el 58,33\% se encontraban en una fase inicial (hasta tres años en situación de calle) del proceso de exclusión social, el $25 \%$ en fases avanzadas (entre tres y cinco años) y el $16,66 \%$ en fases consolidadas (más de cinco años). Entre los factores facilitadores en fases iniciales destacan la importancia de disponer de apoyos institucionales y sociales, el acceso a un trabajo y a una vivienda y la capacidad de afrontamiento frente a las adversidades. Diversos autores afirman que articular recursos y apoyos sociales en momentos críticos de exclusión social previene encontrarse en situaciones de calle (Sarasa y Sales, 2009; Sánchez Morales, 2010). En fases avanzadas y consolidadas destacan como elementos favorables los apoyos institucionales que acompañan a largos procesos de convalecencia y los apoyos estructurales vinculados a los sistemas de protección social. Un elemento favorable en todas las fases es disponer o recuperar redes de apoyo que garanticen los procesos de recuperación personal y social de diversa intensidad.

Los factores que dificultan en todas fases se vinculan con las limitaciones que presentan los sistemas de protección (en relación con el acceso a la vivienda, a la inserción laboral y a la garantía de ingresos 
en situaciones de exclusión social, así como a los apoyos institucionales en momentos claves del proceso de inclusión social) y la debilitación de las redes sociales. Tal como afirman diversos autores (Gracia Fuster et al., 1995; Muñoz et al., 2003) la ausencia o pérdida del capital social genera consecuencias negativas en la salud general y especialmente pueden afectar a la salud mental.

“Estoy en un piso compartido donde la convivencia con los compañeros es muy buena. Somos como una pequeña familia...Por el tema de la adicción, estoy siguiendo un tratamiento en el CAS [Centro de Atención y Seguimiento a las Drogodependencias] y recientemente me han diagnosticado un trastorno bipolar de la personalidad. Ahora estoy pendiente de ir a un centro de día por el tema de la salud mental. Y bien, como te decía, ir avanzando poco a poco" (E3).

El estudio realizado por Uribe y Alonso (2009) nos indica que el $49 \%$ de las personas sin hogar encuestadas en la ciudad de Barcelona padecían algún tipo de trastorno mental (depresión, ansiedad o algún tipo de adicciones asociadas). Tal como nos indican diversos autores (Cabrera, 1998; Muñoz et al., 2003; Tezanos, 2004) el mayor tiempo en la calle y la falta de una vivienda propia pueden agravar o desencadenar estos tipos de trastornos. Bachiller (2014), a través de un análisis etnográfico de personas sin hogar en la ciudad de Madrid, nos aproxima a las dificultades de reversión de estos trastornos, asociados con la habituación de la vida en la calle y con la falta de servicios adaptados a las características de los procesos de exclusión social.

Las expectativas de futuro explicitadas por todas las personas sin hogar entrevistadas en esta investigación hacen referencia, en primer lugar, a tener una vivienda propia (manifestadas por todas las personas entrevistadas) y en segundo lugar (un 83,33\% de las personas) a recuperarse de las diversas dificultades de salud. Tras estas expectativas, un 58,33\% de las personas manifiestan querer "mantener o encontrar un trabajo" como la forma más evidente de salir de la situación de sinhogarismo.

Tal como indica la encuesta del INE (2012) el $45 \%$ de las personas se quedaron sin hogar por la pérdida del trabajo. Este dato nos habla de una realidad que se asocia a la crisis económica y a las desigualdades del actual sistema productivo, donde el acceso o la reincorporación al mercado laboral están siendo especialmente difíciles para un gran número de personas. Tal como apuntan Subirats et al. (2009), el acceso es especialmente problemático para algunos colectivos, como la población extranjera o los grupos o colectivos sociales que por sus características encuentran aún más barreras de acceso. En esta línea, un documento de la Comisión Europea (2013) apunta que la inserción laboral de los sin techo es un proceso largo y complejo, dado que muchos de ellos presentan inconvenientes asociados a la salud, a la salud mental o a la baja cualificación académica. En este sentido, se hace especialmente necesaria la organización de actividades o programas orientados a la formación e inserción laboral desde las entidades sociales que atienden a las personas sin hogar.

\subsection{Acompañando procesos de inclusión}

\subsubsection{Los elementos que favorecen y dificultan los procesos de inclusión social de las personas sin hogar}

Los profesionales entrevistados manifiestan que los factores que favorecen los procesos de inclusión social se vinculan a dos dimensiones, una relacionada con las políticas sociales y la otra con las personas atendidas. En relación con las primeras, se destacan como más importantes disponer de recursos y servicios adecuados a los procesos que presentan las personas, contar con un trabajo en red que posibilite la coordinación entre los servicios implicados y realizar acompañamientos centrados en las capacidades y potencialidades de las personas. Y con relación a las segundas, destacan las habilidades y actitudes de las personas acompañadas, las redes sociales con las que cuenta, los hábitos laborales y relacionales de los que dispone, así como su capacidad para afrontar nuevas dificultades (resiliencia).
"Nosotros somos los responsables de buscar las máximas herramientas posibles para que ellos puedan avanzar. Hay una responsabilidad de los profesionales. Por otro lado, sí que pienso que las personas llegan aquí con una historia de vida y también de experiencia con otros servicios. $A$ veces nos llegan personas que vienen de la prisión, de un largo recorrido de calle. 0 sea, ellos ya tienen un bagaje previo. Las capacidades que hayan tenido en el pasado condicionarán cómo vayan respondiendo. Yo creo que los factores son de las dos partes... del profesional, cómo nos situamos, del equipo, cómo somos capaces de reconvertir una situación... y, finalmente, de las personas, que irán respondiendo en función de cómo trabajemos con ellas" (ETS8).

Entre los principales factores que dificultan los procesos de inclusión social destacan: la fragmentación de los servicios de protección social, las limitaciones y prolongación en el acceso a ciertos servicios o prestaciones ocasionados por los recortes en áreas fundamentales como la salud, los servicios sociales o la formación y la inserción laboral. Otro de los factores que inciden negativamente son los problemas de salud mental, para los que los profesionales no cuentan con la formación o los servicios adecuados para poder realizar un acompañamiento en función de sus procesos. En relación con las limitaciones o dificultades de las personas acompañadas, se destacan aquellas situaciones más cronificadas, en las que no se 
pueden alcanzar cambios a pesar de contar con servicios y profesionales a su lado. De manera frecuente, estas situaciones (menores en porcentaje, pero de mayor complejidad en la intervención) van pasando por diversos servicios sociales y sanitarios sin poder conseguir una mejora de su situación. En este sentido, se remarcan las diferencias de género con relación a estas situaciones más deterioradas. Los profesionales afirman que las mujeres tienen una representación menor, pero están más afectadas a nivel de salud y a nivel emocional en relación con los hombres.

La situación de crisis actual puede incidir negativamente en los procesos de inclusión social de las personas sin hogar. Según Vernia (2011) el Síndic de Greuges de Catalunya denunciaba un fuerte crecimiento durante el año 2011 de las quejas relacionadas con los derechos en los ámbitos de la salud, la educación o los servicios sociales en personas y familias que se encontraban en una situación de pobreza o en un proceso de exclusión social. Los procesos de inserción se alargan debido a limitaciones en el acceso (como es el caso de la renta mínima de inserción), o a falta de plazas en algunos recursos de la red de servicios sociales (pisos de personas mayores) o de salud mental (servicios intermedios como centros de día).

Los factores que dificultan los procesos en relación con el género se relacionan con condicionantes de tipo cultural.

"Sí, esta residencia es mixta. Tenemos pocas mujeres, pero las que están se encuentran en una situación más límite. Es más extrema que la de los hombres. Están más afectadas físicamente y emocionalmente. Además, están doblemente estigmatizadas, por ser personas sin hogar y además ser mujeres. Se les penaliza mucho más culturalmente y ellas se sienten mucho más culpables y están más machacadas familiarmente que los hombres" (ETS2).

Tal como afirma una de las trabajadoras sociales entrevistadas, las mujeres se encuentran doblemente estigmatizadas, por encontrarse sin hogar y por ser mujeres. En esta línea, diversos autores (Escudero, 2003; Bernard, 2010; Morata et al., 2012) hablan de mayores desigualdades materiales (vinculadas al mercado laboral), institucionales (configuración de servicios) y de niveles más altos de victimización (relacionados con la violencia doméstica).

\subsubsection{El conjunto de actuaciones profesionales que intervienen en los procesos de inclusión de las personas sin hogar: el acompañamiento social}

Los resultados de las entrevistas realizadas a los trabajadores sociales nos plantean que el acompañamiento social es la forma de intervención más adecuada en procesos de inclusión social del sinhogarismo. Diversos documentos avalan estas conclusiones (FEPSH, 2013; Rais ,2007; Red de Lucha contra la pobreza y la exclusión social, 2011), poniendo el acento en una intervención de proximidad proactiva e intensiva. Las premisas que destacan en esta forma de intervención se basan en la vinculación y el compromiso compartido como base fundamental de la relación establecida entre acompañados y acompañantes.
"Yo soy TS [trabajadora social] de profesión y de vocación. Lo que hacemos en trabajo social es primero fortalecer mucho la vinculación. Más con este tipo de población que viene de vuelta de todo. La media es que hayan estado más de cinco años en calle. Si no hay confianza y vínculo. Una de las claves de la entidad es tener mucha conciencia de con quién trabajamos y los límites que tenemos. Si no tenemos seguridad en nuestra vida, no acaba de funcionar el tema. Hay personas que están vinculados con nosotros desde hace muchos años" (ETS2).

Según diversos documentos (Fepsh, 2013; Rais, 2007; Red de Lucha contra la pobreza y la exclusión social, 2011) el acompañamiento social es un método de intervención profesional intensivo y continuado que se caracteriza por ser proactivo y estar centrado en las capacidades y potencialidades de las personas atendidas. El trabajador social se convierte en un acompañante del viaje ofreciendo nuevas opciones y ayudando a descartar las metas inviables (De Robertis, 1992; Rais, 2007; Raya y Caparrós, 2014). A través de la escucha activa y del respeto se establecerán las bases de la relación entre acompañados y acompañantes.

Diversos autores (Cabrera, 2008; Roca, 2011; Tezanos, 2004) afirman que el sinhogarismo requiere de intervenciones integrales que avalen la necesidad de designar a los profesionales de las entidades sociales como responsables y referentes de estos casos complejos. El objetivo final del acompañamiento social realizado con las personas sin hogar es conseguir su participación activa fomentando de esta forma la promoción de una ciudadanía inclusiva. En este sentido, otra trabajadora social entrevistada nos introduce al concepto de la participación activa.

\footnotetext{
“Una de ellas es el respeto a la persona. Porque cuando la persona se siente respetada puede participar y sentirse parte. Es decirle 'Tú eres el protagonista’. Cuando tú les tratas con respeto, es recíproco. Reforzar las capacidades, la autoestima. Siempre se ha de hacer refuerzo positivo. Y muchos descubren cosas que no sabían de ellos mismos" (ETS4).
}

En este sentido, el acompañamiento social ha de centrarse en el reconocimiento de los derechos que tienen de todos los ciudadanos a recibir una atención personalizada y mantenida en el tiempo. Con relación a las formas de actuación profesional, se preguntaba a los trabajadores sociales sobre los modelos y técnicas utilizadas en los procesos de 
acompañamiento social con las personas sin hogar. Los trabajadores sociales entrevistados se han situado próximos al modelo humanista.

“El modelo con el que más me identifico es el centrado en la persona. Lo que hacemos mucho es trabajar con la autonomía y las capacidades de las personas. Este sería el punto de partida del trabajo como trabajadores sociales. No debemos centrarnos en los problemas, sino en sus habilidades y en cómo facilitar que la persona las desarrolle y aprenda a ver sus potencialidades. Y si las acciones que hacemos no funcionan que, estas puedan ser vistas como oportunidades y no como fracasos. Por ejemplo, nosotros en Programa de pisos le damos la responsabilidad a la persona. ¿Cuándo puedes hacer todo el proceso? ¿Cuándo quieres marchar del piso? Nosotros evidentemente estamos para acompañarlos, pero en un segundo plano" (ETS5).

Tal como afirma Viscarret (2007), desde este modelo destacan valores como el respeto, la tolerancia y la ausencia de directividad con la persona acompañada. Los profesionales posicionados desde este modelo se convertirán, en palabras de Howe (1999) en "buscadores de sentido", ayudando a través del espacio del acompañamiento social a comprender y compartir proyectos vitales de larga duración y de importante intensidad. Sin embargo, algunos trabajadores sociales entrevistados han tenido dificultades en identificar el modelo teórico aplicado en las intervenciones sociales.

En los resultados obtenidos sobre las técnicas más utilizadas por los trabajadores sociales que intervienen con personas sin hogar destacan, en primer lugar, las vinculadas a la intervención individual de gestión del caso, como la observación, la entrevista (de acogida, seguimiento y valoración) y la planificación (codiagnóstico, plan compartido con la persona acompañada, tramitación, gestión y coordinación de recursos y evaluación). Desde una perspectiva integral, el trabajador social realiza una acogida, valoración y acompañamiento social adecuados a las características del caso, aplicando técnicas basadas en la relación y mediación de procesos de exclusión social de diversa intensidad. En segundo lugar, destacan las vinculadas a la intervención grupal con personas sin hogar (de carácter socioeducativo) y las vinculadas al trabajo entre profesionales (interdisciplinares y multidisciplinares). Las actividades grupales realizadas con personas sin hogar están orientadas a la socialización, a la rehabilitación, a la mejora de hábitos, a la inserción laboral y a la convivencia entre personas alojadas en diversos tipos de alojamientos. Y, en tercer lugar, destacan las técnicas vinculadas a la intervención comunitaria, entre las que se encuentran el trabajo en red y las actividades orientadas a la sensibilización. En el primer caso, se hace necesaria la articulación de un trabajo coordinado y consensuado entre las entidades y profesionales que trabajan en la
XAPSLL y los diversos sistemas de protección social implicados. Siguiendo los diferentes niveles de construcción de la red propuestos por Rovere (1999) vemos que la mayoría de las entidades se encuentra ubicada en los tres primeros niveles (asociarse, cooperar, colaborar) y quedan por incorporar los otros dos niveles (conocer y reconocer) que suponen una mayor consolidación y confianza entre los servicios implicados. En el segundo caso, las entidades realizan una labor destacada de información y sensibilización sobre el sinhogarismo que disminuye la fuerte estigmatización hacia estas situaciones y genera una sociedad más justa y solidaria. La reciente implementación del modelo Housing First está requiriendo de la aplicación de técnicas evaluativas de procesos que serán de gran utilidad para avanzar en la mejora de la práctica profesional.

En nuestro país existe escasa bibliografía sobre el espacio establecido entre los acompañados y los acompañantes. La investigación social sobre personas sin hogar se ha centrado en conocer las causas y efectos del sinhogarismo, así como las políticas sociales que se han llevado a cabo. Aún queda mucho por conocer en relación con las formas de intervención realizada por los trabajadores sociales a lo largo de los diversos y complejos procesos de inclusión social por los que transitan las personas sin hogar.

\section{Conclusiones y propuestas}

Los procesos de inclusión social de las personas sin hogar son atravesados por diversos factores protectores que se vinculan a los ámbitos estructural, institucional, relacional y personal planteados por diversos autores (Cabrera, 2008; Tezanos, 2004; Subirats, 2004). Entre los factores protectores destacan la disponibilidad de programas y recursos orientados a los procesos, el acompañamiento social intensivo y transversal, y disponer de estrategias resilientes y de redes de apoyo. Entre los factores que limitan destacan las desigualdades sociales y educativas, la debilidad del sistema de protección social, carecer de apoyos sociales y presentar diversas dificultades asociadas a la salud.

La intervención profesional aplicada en los procesos de inclusión social con personas sin hogar se basa en la metodología del acompañamiento social. Diversos documentos avalan esta afirmación (FEPSH, 2013; Rais, 2007; Red de Lucha contra la pobreza y la exclusión social, 2011), poniendo el acento en una intervención de proximidad proactiva e intensiva. Los trabajadores sociales realizan un acompañamiento social que se caracteriza por ser proactivo, intensivo y transversal. El modelo más utilizado es el humanista y las técnicas utilizadas se vinculan a la intervención individual (gestión del caso), a la intervención grupal (a través de grupos socioeducativos) y a la intervención comunitaria (a través del trabajo en red y la sensibilización). 
Las recomendaciones finales de esta investigación se centran en dos grandes áreas: las políticas sociales y la intervención social. En relación con las primeras, es necesario articular propuestas orientadas a la regulación y al acceso a la vivienda, diseñar planes regionales y locales contra el sinhogarismo, implementar servicios en clave de proceso e introducir la perspectiva de género en el diseño de las políticas sociales. Y en relación con las segundas, es necesario implementar actuaciones preventivas desde los territorios, evaluar procesos y proponer nuevas estrategias de intervención, articular actuaciones y programas conjuntos con otros servicios, incrementar las actuaciones grupales y comunitarias y realizar investigaciones conjuntas entre el espacio académico y el profesional. 


\section{Referencias bibliográficas}

AYUNTAMIENTO DE BARCELONA (2007): Programa municipal de atención a persones sin techo.

ANDERSON, I. y CHRISTIAN, J. (2003): “Causes of Homelessness in the UK: A Dynamic Analysis", Journal of Community Applied Social Psychology, 13, págs. 105-118.

BAUMAN, Z. (2000): Modernidad líquida, Argentina, Fondo de Cultura Económica.

BECK, U. (1998): La sociedad del riesgo: hacia una nueva modernidad, Barcelona, Paidós.

BERNARD, N. (2010): "The gender of housing deprivation in Belgium", Homeless in Europe, Spring 2010, págs. 24-26.

BUSCH-GEERTSEMA, V. (2013): Housing First Europe: Final Report, Bruselas/Bremen, Progress.

BUSQUET, J.; MEDINA, A. y SORT, J. (2006): La recerca en comunicació: Què hem de saber? Quins passos hem de seguir?, Barcelona, Universitat Oberta de Catalunya.

CABRERA, P.J. (1998): Huéspedes del aire. Sociología de las personas sin hogar en Madrid, Madrid, Universidad Pontificia Comillas.

- y RUBIO, M.J. (2008): “Las personas sin hogar, hoy", Revista del Ministerio de Trabajo e Inmigración, 75, Madrid, pp. 51-74.

CASTEL, R. (1991): “De l'indigence à l'exclusion, la désaffiliation. Précarité du travail et vulnérabilité relationnelle", en DANZELOT, J.: Face à l'exclusion. Le modèle français, París, Esprit.

CASTEL, R. (1997): La metamorfosis de la cuestión social. Una crónica del asalariado, Buenos Aires, Paidós.
COMISIÓN EUROPEA (2013): Confronting Homelessness in the European Union, Bruselas, SWD.

DECLERCK, P. (2001): Les naufragés. Avec les clochards de Paris, París, Terre

Humaine, Plon.

DE ROBERTIS, C. (1992): Metodología de la intervención en Trabajo Social, Barcelona, El Ateneo.

EAPN (2013): El Modelo Housing First, una oportunidad para la erradicación del

sinhogarismo en la Comunidad de Madrid. Recuperado de 〈http://eapnmadrid.org/?pagina=documentos \&seccion $=15$.

ERANSUS, B. (2004): “El acompañamiento como herramienta de lucha contra la exclusión", Documentación, 135, págs. 91-106.

ESCUDERO, M.J. (2003): Mujeres sin hogar en Granada. Un estudio etnográfico,

Granada, Universidad de Granada.

FEANTSA (2009): European Review of Statistics on Homelessness, Bruselas, Feantsa.

- (2013): On the Way Home? Monitoring report on Homelessness and Homeless Policies in Europe, Bruselas, European Federation of National Organisations working with the Homeless, AISBL.

FEANTSA y OSW (2005): Auditoria sobre participación de Feantsa y Osw. Involucrar a las personas sin hogar en la toma de decisiones que afectan a los servicios de los que son usuarios: Una visión de conjunto de las prácticas existentes entre las entidades que trabajan con este colectivo, Bruselas, Feantsa. 
FEDERACIÓN DE ENTIDADES DE APOYO A LAS PERSONAS

SIN HOGAR (2013): Documento marco de la Federación de Entidades de apoyo a las personas sin hogar, Madrid, FEPSH.

FUNDACIÓN RAIS Y ASOCIACIÓN REALIDADES (2007): Construyendo relaciones. Intervención psicosocial con personas sin hogar, obtenido de 〈http://www.carm.es/ctra/cendoc/ haddock/13374.pdf〉.

FUNES, J. (2001). La nova llei penal i educativa: una lectura en clau educativa i que intenta ser il.lusionada. Educació social, 18. Barcelona, maigagost,2001.

- et al. (2001): El acompañamiento y los procesos de incorporación social, Guía para su práctica. Federación Sartu, Dirección de Bienestar Social, Gobierno Vasco.

- y ALONSO, A. (2009): "Transiciones, itinerarios y procesos", Educación Social: Revista de Intervención Socioeducativa, 42, págs.15-27.

GARCÍA ROCA, J. (2006): "Relatos, metáforas y dilemas para transformar las exclusiones", en $V$ Informe EUHEM de Políticas Sociales. La exclusión social y el Estado de Bienestar en España, VIDAL FERNÁNDEZ, F. (ed.), Madrid, Fuhem.

GRACIA FUSTER, E.; HERRERO OLAIZOLA, J. y MUSITU OCHOA, G. (1995): El apoyo social, Barcelona, PPU.

HOWE, D. (1997): La teoría del vínculo afectivo para la práctica del trabajo social, Barcelona, Paidós.

INSTITUTO NACIONAL DE ESTADÍSTICA (2012): Encuesta a las personas sin hogar de 2011.

MORATA, B.; MORANTE, M. y TRUJILLO, M. (2010): “I Seminario Interdisciplinar sobre género y sexualidad en el siglo XXI: política afectivosexual, migraciones femeninas y vulnerabilidad social”, Revista Hispania, I (2), Universidad de Granada Trabajo Social Global, págs. 242-262.

MORENO-MÁRQUEZ, G. (2012): “Exclusión social severa y sinhogarismo. ¿Qué opinan las personas usuarias sobre los recursos?", Portularia, XII, número extra 2012, IX Congreso Nacional de Facultades de Trabajo Social, Universidad de Huelva Publicaciones, págs. 245-253.

MUÑOZ, M. y VÁZQUEZ, C. (1998): “Las personas sin hogar: aspectos psicosociales de la situación española”, Intervención psicosocial, 7 (1), págs. 7-26.

- y PANADERO, S. (2004): “Intervención con personas sin hogar con trastornos mentales graves y crónicos en Europa", Rehabilitación psicosocial, 1 (2), págs. 64-72.

-; PANADERO, S.; PÉREZ SANTOS, E. y QUIROGA, M.A. (2005): "Role of stressful life events in homelessness: An Intragroup analysis", American Journal of

Community Psychology, 35(1/2), págs. 35-47.

RAYA, E. y CAPARRÓS, N. (2014): “Acompañamiento como metodología de Trabajo Social en tiempos de cólera", Cuadernos de Trabajo Social, 27-1, págs. 81-91.
RED NAVARRA DE LUCHA CONTRA LA POBREZA Y LA EXCLUSIÓN SOCIAL (2011): El acompañamiento como método de intervención en los procesos de inclusión, Pamplona, Red Navarra.

RENES, V; FUENTES, P.; RUIZ, E. y JARAíZ, G. (2007): "Realidad, pensamiento e intervención social", Documentación Social. Revista de Estudios Sociales y de Sociología aplicada, 14, págs. 11-35.

RUIZ, J.I. (2007): Metodología de la investigación cualitativa, Bilbao, Universidad de Deusto.

ROVERE, M. (1999): Redes en salud; un nuevo paradigma para el abordaje de las organizaciones y la comunidad, Rosario, Secretaría de Salud Pública/AMR, Instituto Lazarte (reimpresión).

SALES, A. (2013): Diagnosi 2013, Barcelona, Ajuntament de Barcelona, Xapsll.

SÁNCHEZ MORALES, M.R. (2010): “Las personas 'sin hogar' en España”, RES, 14, págs. 21-42.

SARASA, S., SALES, A. (2009). Itineraris i factors d'exclusió social. Barcelona: Síndica de Greuges de Barcelona.

SIEBER, J.E., STANLEY, B. (1988): "Ethical and professional dimensions of socially sensitive research", American Psychologist, vol. 43, págs. 49-55.

SUBIRATS, J. (2004): Pobresa i exclusió social. Una anàlisi de la realitat espanyola i europea, Barcelona, Fundació "La Caixa".

SUBIRATS, J., ALFAMA, E., OBRADORS, A. (2009): "Ciudadanía e inclusión social frente a las inseguridades contemporáneas. La significación del empleo", Documentos de Trabajo, Fundación Carolina, 32.

TEZANOS, J.F. (2004). Tendencias en desigualdad y exclusión social. Tercer Foro sobre tendencias sociales, Madrid, Editorial Sistema.

VÁZQUEZ, J.J., PANADERO, S., IBORRA, A., MARÍN, C. y MARTÍN, R. (2011): “Resultados preliminares del proyecto 'Estudio de la incidencia de los sucesos vitales estresantes, atribuciones causales y estereotipos en los procesos de exclusión inclusión social de las personas sin hogar', Comunicación presentada en el VI Congreso Chileno de Psicología, Santiago de Chile.

VISCARRET, J.J. (2007): Modelos y métodos de intervención en Trabajo Social, Madrid, Alianza editorial.

URIBE, J. (2014): De la calle al hogar. Housing First como modelo de intervención y su aplicación en Barcelona. Barcelona, Ediciones Sant Joan de Déu.

VERNIA, S. (2011): “L’actuació del Síndic de Greuges en el context social de crisi econòmica": Revista de Treball Social (RTS), 194, Barcelona, Col.legi Oficial de Treball Social de Catalunya, págs. 21-25.

VIDAL, F. (2009): Pan y rosas. Fundamentos de exclusión social y empoderamiento. Madrid, Cáritas Española, Foessa. 